\title{
Density and temperature of energetic electrons in the Earth's magnetotail derived from high-latitude GPS observations during the declining phase of the solar cycle
}

\author{
M. H. Denton ${ }^{1}$ and T. E. Cayton ${ }^{2}$ \\ ${ }^{1}$ Department of Physics, Lancaster University, Lancaster, LA1 4YB, UK \\ ${ }^{2}$ ISR-1, Los Alamos National Laboratory, Los Alamos, NM 87545, USA
}

Received: 14 July 2011 - Revised: 20 September 2011 - Accepted: 27 September 2011 - Published: 7 October 2011

\begin{abstract}
Single relativistic-Maxwellian fits are made to high-latitude GPS-satellite observations of energetic electrons for the period January 2006-November 2010; a constellation of 12 GPS space vehicles provides the observations. The derived fit parameters (for energies $\sim 0.1-1.0 \mathrm{MeV}$ ), in combination with field-line mapping on the nightside of the magnetosphere, provide a survey of the energetic electron density and temperature distribution in the magnetotail between McIlwain L-values of $L=6$ and $L=22$. Analysis reveals the characteristics of the density-temperature distribution of energetic electrons and its variation as a function of solar wind speed and the Kp index. The density-temperature characteristics of the magnetotail energetic electrons are very similar to those found in the outer electron radiation belt as measured at geosynchronous orbit. The energetic electron density in the magnetotail is much greater during increased geomagnetic activity and during fast solar wind. The total electron density in the magnetotail is found to be strongly correlated with solar wind speed and is at least a factor of two greater for high-speed solar wind $\left(V_{\mathrm{SW}}=500-1000 \mathrm{~km} \mathrm{~s}^{-1}\right)$ compared to low-speed solar wind $\left(V_{\mathrm{SW}}=100-400 \mathrm{~km} \mathrm{~s}^{-1}\right)$. These results have important implications for understanding (a) how the solar wind may modulate entry into the magnetosphere during fast and slow solar wind, and (b) if the magnetotail is a source or a sink for the outer electron radiation belt.
\end{abstract}

Keywords. Magnetospheric physics (Magnetotail)

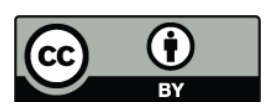

Correspondence to: M. H. Denton (m.denton@lancaster.ac.uk)

\section{Introduction}

Numerous observations of particle populations in the magnetotail have been reported and surveys of these data are available in the literature. However, in the last twenty years the majority of these observations have addressed the lowto-medium energies $(\sim 1 \mathrm{eV}$ to a few tens of $\mathrm{keV})$. Studies of highly energetic electrons $(>100 \mathrm{keV})$ in the magnetotail were carried out relatively early in the space-era (e.g. Montgomery, 1968; Murayama and Simpson, 1968; Meng, 1971; Walker and Farley, 1972) - few recent studies of energetic electron fluxes in the magnetotail have been carried out and apart from the recent work of Borovsky and Cayton (2011), other studies have not focused on the density and temperature of the population. In this study we present a survey of the energetic electron $(0.1-1.0 \mathrm{MeV})$ density and temperature in the Earth's magnetotail. Assuming the flux measured at a single point on a particular field line represents the flux at other points along that field line then measurements from GPS satellite orbits may, under certain conditions, be mapped into the magnetotail and provide information on the region from a spatially distant location. The motivation for this study is to utilise this fact to reveal the spatial and temporal distribution of energetic electrons in the magnetotail, and subsequently how these distributions respond to changes in solar wind and geomagnetic conditions (cf. Denton and Borovsky, 2011).

Electrons with relativistic energies are known to occupy the magnetotail and studies of these electrons have focused on electron "bursts" - short-lived (a few seconds) observations of high fluxes observed in the near- and deepmagnetotail (e.g. Terasawa and Nishida, 1976; Taylor et al., 2006). Such energetic electrons may be assumed to arise from either of two sources, namely (a) as a result of localised energisation processes in the magnetosphere (e.g. 
reconnection events), or (b) via direct entry from the solar wind into the magnetotail.

A survey of over 10 satellite-years of IMP7 and IMP8 measurements (Meng et al., 1981) provided a detailed statistical study of the spatial distribution of energetic particle fluxes in the magnetotail at distances between $\sim 30-40$ Earth radii $\left(R_{\mathrm{E}}\right)$. The study emphasised energetic electrons $(\sim 0.22-2.5 \mathrm{MeV})$ are present in the magnetotail much of the time (not only in "bursts"), and that a positive correlation exists between geomagnetic activity (proxied by $\mathrm{Kp}$ ) and "occurrence frequency" - the occurrence of flux exceeding a particular threshold. This finding is consistent with the earlier study by Murayama and Simpson (1968) who also found evidence of an increase in energetic electron flux in the magnetotail with increasing activity (proxied by $\mathrm{Kp}$ ) using data from the IMP1 satellite. More recently Åsnes et al. (2008) studied the statistical properties of plasma sheet electrons using a limited set of observations from the Cluster spacecraft. They concluded that the intensity of electrons was strongly correlated with the plasma temperature and that a harder spectrum was observed at dawn compared to dusk.

Satellites of the Global Positioning System (GPS) execute $\sim 4.2 R_{\mathrm{E}}$ circular orbits inclined $55^{\circ}$ with respect to the geographic equator; the satellites are distributed among 6 orbit planes, A-F. An increasing number of these satellites carry energetic particle sensors that measure the distribution of energetic electrons in the energy range $0.1-1.0 \mathrm{MeV}$ (Cayton et al., 1998; Distel et al., 1999). Data from 12 satellites during the period January 2006 through November 2010 are used in this study. Relativistic-Maxwellian fits are made to the electron distributions and fit-values of temperature and density are calculated (see also Borovsky and Cayton, 2011). Magnetic field-line tracing from the satellite into the magnetotail is carried out using the T89 model (Tsyganenko, 1989) and the equatorial crossing of the field line is derived. This enables a survey of the energetic electron density and temperature as a function of downtail distance. Major geomagnetic storm activity during this period was rare and magnetospheric convection was generally low (Kp rarely exceeded 4) - hence there are poor statistics to fully investigate storm conditions. However, periods of "fast" solar wind, sourced from coronal holes, frequently occur. These periods are examined and we show that the density in the magnetotail is substantially greater during high-speed solar wind. This result has potential implications for determining source/loss of energetic electrons to/from the outer electron radiation belt; i.e. the magnetotail may act as (a) a reservoir, or (b) a sink, of energetic electrons. Alternatively, these electrons may be signatures of acceleration processes elsewhere in the magnetosphere.

\section{Analysis: a density-temperature description of elec- trons in the magnetotail}

A density-temperature description of the energetic electron population has the potential to provide more information than the more-traditional flux description: the flux changes as a result of either (a) an increase/decrease in the local density, or (b) an increase/decrease in the local temperature. Flux is useful in determining effects on instrumentation whereas density and temperature are the underlying physical parameters. In this study we apply a similar technique to that previously used in analysis of data from geosynchronous orbit. The technique, first implemented by Cayton et al. (1989) and subsequently by Borovsky et al. (1998), Borovsky and Denton (2009), Denton et al. (2010), and Borovsky and Cayton (2011), has proved successful in helping understanding radiation belt dynamics, particularly during high-speed-solarwind-stream (HSS) events.

The Combined X-ray and Dosimeter (CXD) (Distel et al., 1999) instruments now operating on 10 Global Positioning System (GPS) satellites, NS53 through NS62, of the GPS replenishment and follow-on series (identified by the designations, Block IIR and IIF) include two subsystems for monitoring energetic electrons: the Low Energy Particle (LEP) subsystem resolves 0.14 to $>1.25 \mathrm{MeV}$ electrons into 5 deposited-energy channels; the High-energy X-ray and Particle (HXP) subsystem resolves 1.3 to $>5.8 \mathrm{MeV}$ electrons into 6 deposited-energy channels. The collimator used for each subsystem samples a wide field of view while limiting the particle flux incident upon the detector elements themselves to an acceptable level: LEP and HXP monitor the omni-directional electron flux incident upon the GPS space vehicle and record their data in on-board memory for downloading during routine contact with the space vehicle (once per day). Likewise, the Burst Detection Dosimeter (BDDIIR) (Cayton et al., 2010) operating on two earlier Block IIR satellites NS41 and NS48 uses 8 separate silicon detectors in shield/filter/sensor configurations to monitor 01 to $>6 \mathrm{MeV}$ electrons.. The deposited-energy thresholds of every instrument were calibrated carefully and used in the evaluation of response functions for electrons incident upon the channels by detailed modelling (Tuszewski et al., 2002) of the instrument by Monte Carlo methods.

A density-temperature description of the GPS energetic electrons is obtained by a relativistic Maxwellian fit to the observed counting rates. This methodology differs from the approach of Christon et al. $(1988,1989)$ who applied a single functional fit across a broad energy range from $50 \mathrm{eV}$ to $\sim 1 \mathrm{MeV}$ to investigate the spectral characteristics of the plasma sheet. In general a kappa distribution provides a good fit for protons $\sim 1 \mathrm{MeV}$ but for electrons fits tend to be dominated by the $<100 \mathrm{keV}$ population. Above $\sim 200 \mathrm{keV}$ measured fluxes deviate significantly from those predicted by a single functional form. As noted by Mcllwain and Whipple (1986) "Hot plasmas coexist with cold plasmas. 
These different populations interact, but each occupies its own niche in phase space". Hence, rather than fitting a single function across multiple plasma populations (thermal, plasma sheet, outer radiation belt, etc.), we fit a single relativistic Maxwellian to the high energy population of electrons in the energy range $\sim 0.1-1.0 \mathrm{MeV}$. Individual examples of this procedure for particular spectra may be found in Cayton et al. (1989).

Data are processed at Los Alamos National Laboratory as follows. First, the observed counting rates are extracted from the compressed 240-s accumulations, and 8 geomagnetic coordinates are appended to the three geographic coordinates already present in the data file. The magnetic field magnitude, $\boldsymbol{B}$, at the position of the space vehicle is evaluated from the T89 model using the current IGRF internal field and the external field corresponding to planetary activity index $\mathrm{Kp}$ in the range $2-$ to $2+$; next, the magnetic filed line that passes through the satellite is traced from the satellite to its northern and southern foot points on Earth's surface; the position and value of the minimum magnitude $B_{0}$ along this field line is recorded, and the bounce invariant for particles mirroring at the satellite is evaluated; these quantities and Hilton's asymptotic expansion (Hilton, 1971) determine an approximate value (a very good one) for McIlwain's L parameter; two cylindrical coordinates for an equivalent dipole field are also calculated, the distance from the dipole axis, and the distance from the magnetic equatorial plane of the dipole field. Second, the input or true counting rate (the observed counting rate corrected for deadtime) for each channel is modelled as a sum of two contributions: non-electron background counts plus counts that result from a spectrum of incident electrons. (When integrated with the CXD or BDD instrument response functions mentioned above the model spectrum yields predictions for the counting rates in all channels as a consequence of the incident electrons.) Third, bestfit values for the parameters of the model spectrum are inferred by the method of least-squares, minimizing the sum of the squared deviations between the sets of true and predicted counting rates for each data record. Fourth, either integral or differential electron fluxes at standard energies are evaluated from the best-fitting spectrum. The analysis is optimised for the energies $\sim 0.1-1.0 \mathrm{MeV}$ and differential energy fluxes are calculated at five energies within this range. The final fits are valid for L-values greater than 6 .

Whilst electrons in the near-Earth (trapping) region are anisotropic to some degree (e.g. Fritz et al., 2003; Borovsky and Denton, 2011) the method of mapping GPS measurements described here is considered valid given numerous previous observations which indicate the electrons are quasiisotropic in the more distant magnetotail (e.g. Hones et al., 1968; Retzler and Simpson, 1969; Sarris et al., 2006). When the distributions are not quasi-isotropic it is taken as an indication that the spacecraft are on open field lines (e.g. Baker and Stone, 1976). Calculations of the individual pitch angles for the data used in the analysis are shown in Fig. 1 and

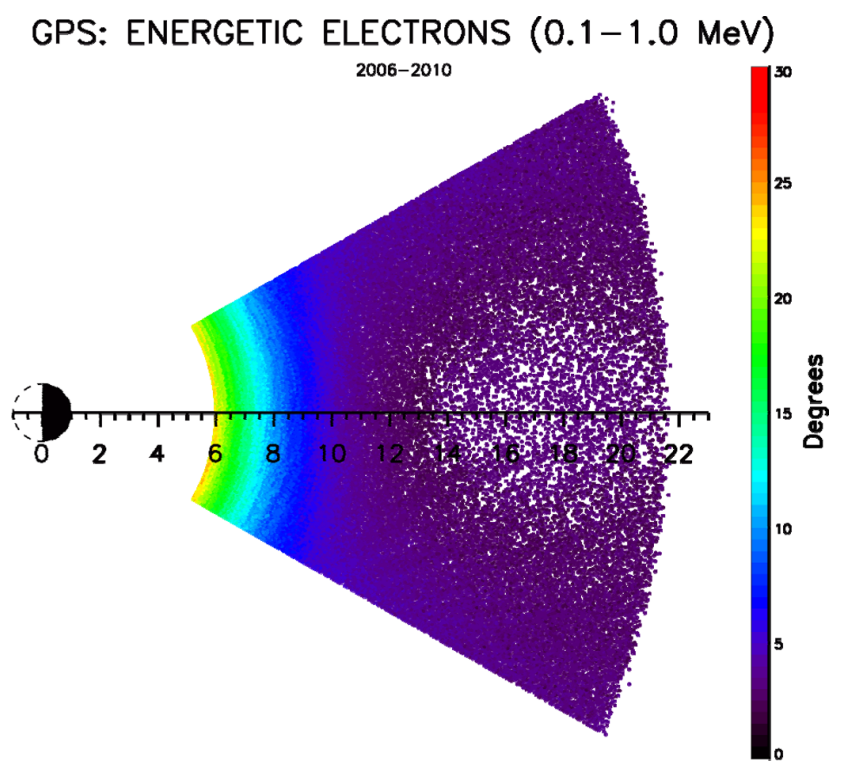

Fig. 1. Scatter plots of the pitch angle of the electrons derived from measurements by GPS spacecraft at high-latitude and then mapped to the equatorial plane using the Tsyganenko T89 model as a function of LT and L. Substantial overlap of the 151574 data points occurs in the region closest to Earth.

indicate that the range of pitch angles encountered at GPS altitudes is $\sim 20^{\circ}$ at $L=6, \sim 10^{\circ}$ at $L=8$, and $<5^{\circ}$ at distances of $L>10$, consistent with the results of Klida and Fritz (2009). Isotropy or quasi-isotropy deeper in the magnetotail allows the energetic electron population to be seen and measured at GPS altitudes in a similar way that the ion plasma sheet is seen and measured by low-altitude DMSP spacecraft (e.g. Wing and Newell, 1998; Johnson and Wing, 2009).

Results presented below are restricted to GPS measurements that are made whilst on magnetic field-lines within two hours of local midnight (between 22:00 LT and 02:00 LT) and with McIlwain-L values between 6 and 25. As mentioned above, the downtail distance is calculated by mapping with the T89 model (with a Kp=2 input). This will be at best an approximation to the actual the field line on which the observations are made since the magnetic field is not static in time, even if the solar wind driving is steady. However, the T89 model field under "average" conditions is suitable for the statistical studies carried out here since it is in some sense an average approximation to the actual field which may change rapidly in time.

\section{Results and discussion}

The density and temperature of the energetic electron population in the energy range $\sim 0.1-1.0 \mathrm{MeV}$ is measured by 12 satellites in the years 2006 to 2010. A total of almost 


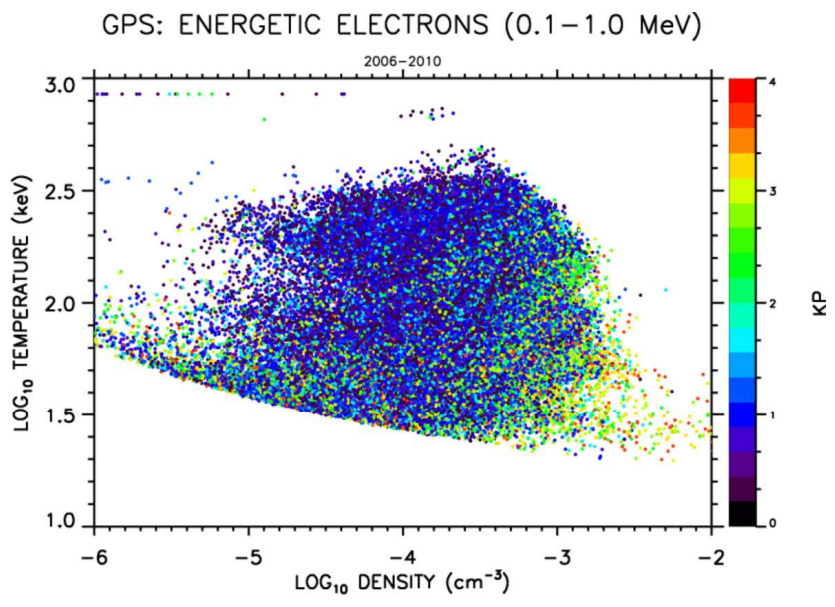

GPS: ENERGETIC ELECTRONS (0.1-1.0 MeV)

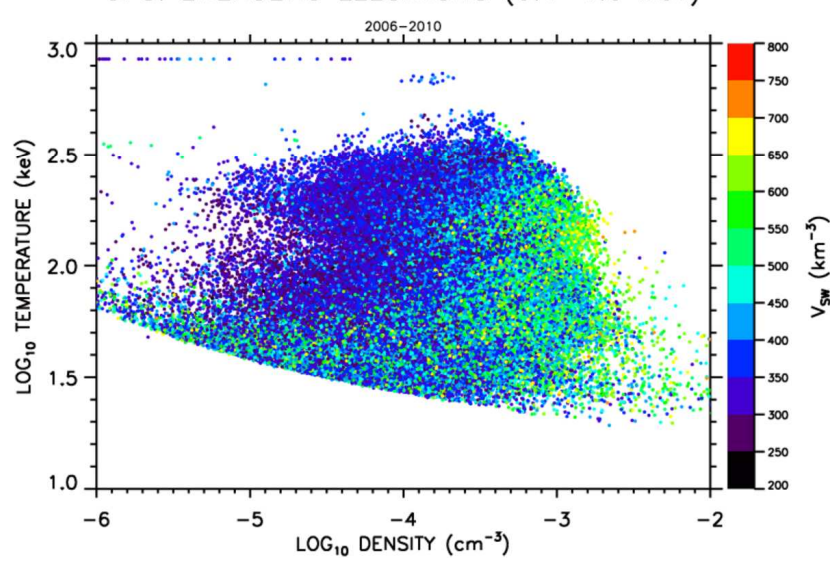

Fig. 2. Scatter plots of the derived density $(N)$ and temperature $(T)$ as a function of the Kp index (top panel) and the solar wind speed (bottom panel) for energetic electrons $(\sim 0.1-1.0 \mathrm{MeV})$ in the magnetotail at local times 22:00-02:00 LT (151 574 data points).

4 million individual data points are examined (all local times) and subsets of these data are plotted below.

Figure 2 contains scatter plots of the energetic electron density (horizontal axis) and temperature (vertical axis) as a function of the derived Kp index (top panel) and as a function of the solar wind speed (bottom panel), with the speed taken from the OMNI2 database (King and Papitashvili, 2005). The mean, variance, skewness, and kurtosis of the logarithm of density and temperature are contained in Table 1 and provide the characteristics of the population studied. The mean value of density and temperature are very similar to those found in the outer electron radiation belt (cf. Denton et al., 2010). Since the majority of the GPS data are inferred to map to the region between $L=6$ and $L=8$, this gives us some measure of confidence that the fitting technique is robust. The density-temperature distribution shown in Fig. 2 (both panels) indicates the temperature of energetic electrons varies by $\sim 1$ order of magnitude and the density varies over $\sim 2$ orders of magnitude. The distribution shows a tendency
Table 1. The mean, variance, skewness, and kurtosis of the logarithm of density and temperature 151574 data points values for the data plotted in Figs. 2 and 3. The mean Kp index for these data is 1.85 and the mean $\mathrm{AE}$ index is 99.

\begin{tabular}{lrr}
\hline & \multicolumn{2}{c}{ All data } \\
\cline { 2 - 3 } & $\mathrm{LOG}_{10} N$ & $\mathrm{LOG}_{10} T$ \\
\hline Mean & -3.72 & 2.00 \\
Variance & 0.28 & 0.08 \\
Skewness & -0.88 & -0.13 \\
Kurtosis & 1.91 & -1.07 \\
\hline
\end{tabular}

to higher density values with increasing $\mathrm{Kp}$ and with increasing solar wind speed. This result is complementary to the findings of Meng et al. (1981) who observed higher fluxes in the more distant magnetotail at $\sim 30-40 R_{\mathrm{E}}$ during enhanced activity (proxied by Kp only). The period 2006-2010 was characterised by generally "calm" conditions - solar and geomagnetic activity were low and the largest possible values of $\mathrm{Kp}$ (which occur around solar maximum) are absent from this analysis (mean $\mathrm{Kp}=1.85$; mean $\mathrm{AE}=99$ ).

Figure 3 contains scatter plots of the energetic electron density (top panel) and temperature (bottom panel) derived from measurements by GPS spacecraft at high-latitude and then mapped to the $\mathrm{x}-\mathrm{y}$ plane using the Tsyganenko T89 model. (Note: significant overlap of the 151574 points occurs for data closest to the Earth). The plots show the spatial extent of the observations (as a function of $\mathrm{L}$ and $\mathrm{LT}$ ), colour-coded by the density (top panel), temperature (middle panel), and count statistics (bottom panel). Data associated with the outer electron radiation belt are evident at L-values between $\sim 6$ and 8 . Further tailwards large density and temperature variations are apparent. There is also evidence for enhanced temperature and density in the post-midnight sector at $\mathrm{L}$ values $>\sim 12$ (although we do not investigate this further in the current study). In order to explore the overall density-temperature variations as a function of the solar wind speed we bin each of the data points into one-hour local-time bins and bins of width one $\mathrm{L}$, and plot the average value. Figure 4 contains the results of this analysis for conditions of (a) $V_{\mathrm{SW}}=100-400 \mathrm{~km} \mathrm{~s}^{-1}$ (left column: 58857 data points), and (b) $V_{\mathrm{SW}}=500-1000 \mathrm{~km} \mathrm{~s}^{-1}$ (right column: 23087 data points). The log of the number of data points for slow and fast wind is shown in the bottom row. A comparison between fast and slow conditions reveals clear differences. The average density of energetic electrons is substantially greater during high-speed solar wind (by at least a factor of 2) in comparison, the magnetotail appears largely "empty" of energetic electrons during slow solar wind. In contrast the temperature of energetic electrons is slightly higher for slow solar wind than for fast solar wind. Fast solar wind is known to be strongly correlated with higher fluxes and higher density in the outer electron radiation belt at geosynchronous 

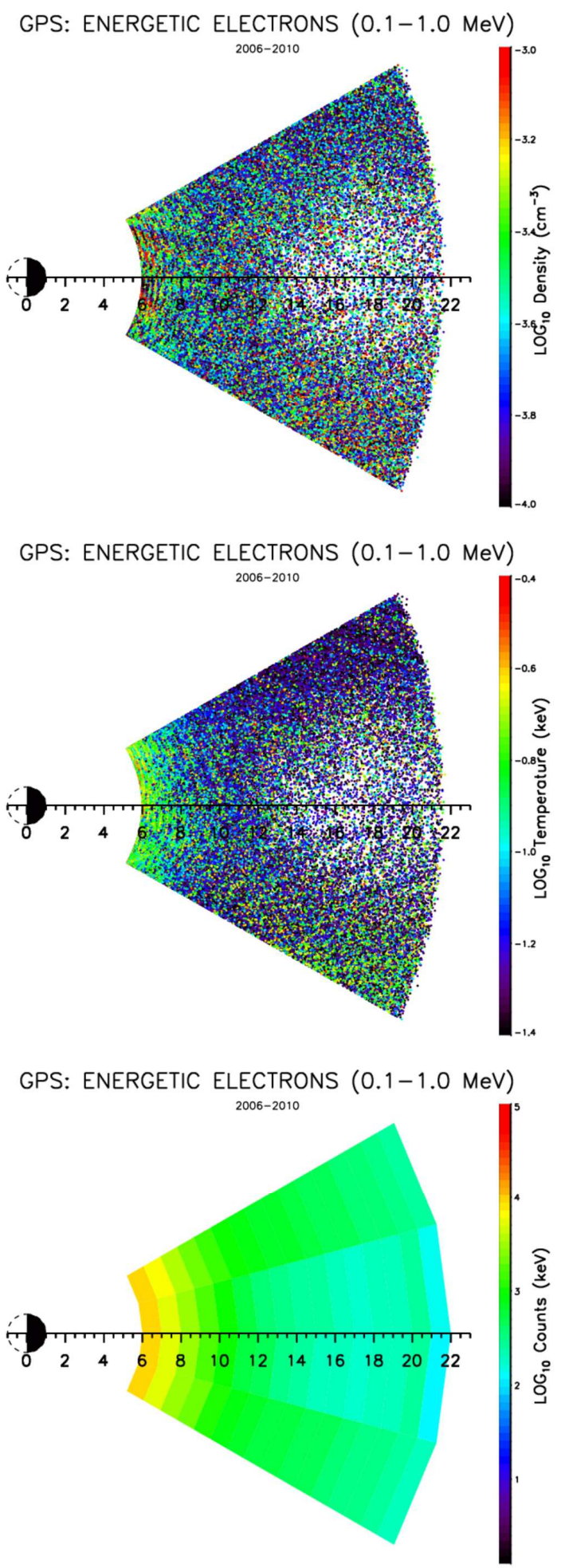

Fig. 3. Scatter plots of the electron density $(N)$ (top panel), electron temperature $(T)$ (middle panel) and counts (bottom panel) derived from measurements by GPS spacecraft at high-latitude and then mapped to the equatorial plane using the Tsyganenko T89 model as a function of LT and L. Substantial overlap of the 151574 data points occurs in the region closest to Earth. orbit, particularly during HSS-events (Borovsky et al., 1998; Borovsky and Denton, 2009; Denton et al., 2010). Our analysis shows that the density of energetic electrons in the magnetotail follows this trend. Note: there are fewer data present in the region very close to local midnight (compared with further dawnwards/duskwards). This is due to stretching of the magnetic field in the central plasma sheet - field lines from GPS altitudes on occasion map to the far magnetotail ( $L>22$ ) meaning fewer data in the region close to local midnight.

The results plotted in Fig. 5 show a similar analysis to that summarised in Fig. 4 however on this occasion the data are filtered with respect to the Kp index. Average energetic electron density and temperature are shown for calm geomagnetic conditions conditions $(\mathrm{Kp}<2$ : left column) and disturbed geomagnetic conditions ( $\mathrm{Kp}>3$ : right column). The plots reveal that the density in the magnetotail is elevated during disturbed conditions with the density more than a factor of two higher on average during elevated activity - for particular events the density may be over an order of magnitude higher during disturbed conditions.

The observations presented in Figs. 2-5 raise certain questions. (i) What is the physical origin of energetic electrons in the magnetotail? A proportion of the electrons may be generated in short-lived bursts (e.g. Terasawa and Nishida, 1976; Taylor et al., 2006). However there is also a population of energetic electrons in the magnetotail which substantially increases in density during fast solar wind. Are these electrons energised in situ or present due to transport from other regions? (ii) Since values of density and temperature of energetic electrons inferred in this study are so similar to those found in the outer electron radiation belt, is the more-distant magnetotail a significant source/sink for the radiation belt?

\section{Summary and future work}

We have presented analyses of energetic $(\sim 0.1-1.0 \mathrm{MeV})$ electron data from the magnetotail and derived the density and temperature of this population for a variety of conditions in the period 2006-2010. For this period of, in general, calm and quiescent geomagnetic conditions we have demonstrated that the energetic electron density and temperature are directly modulated by the solar wind. The findings of this study can be summarised as follows:

1. GPS observations at high latitude can be used to infer the energetic electron density and temperature in the Earth's magnetotail. The density-temperature description provides a robust method for examining energetic electrons in this region. The characteristic density and temperature are similar to those found in the outer electron radiation belt at geosynchronous orbit (cf. Borovsky and Cayton, 2011). 

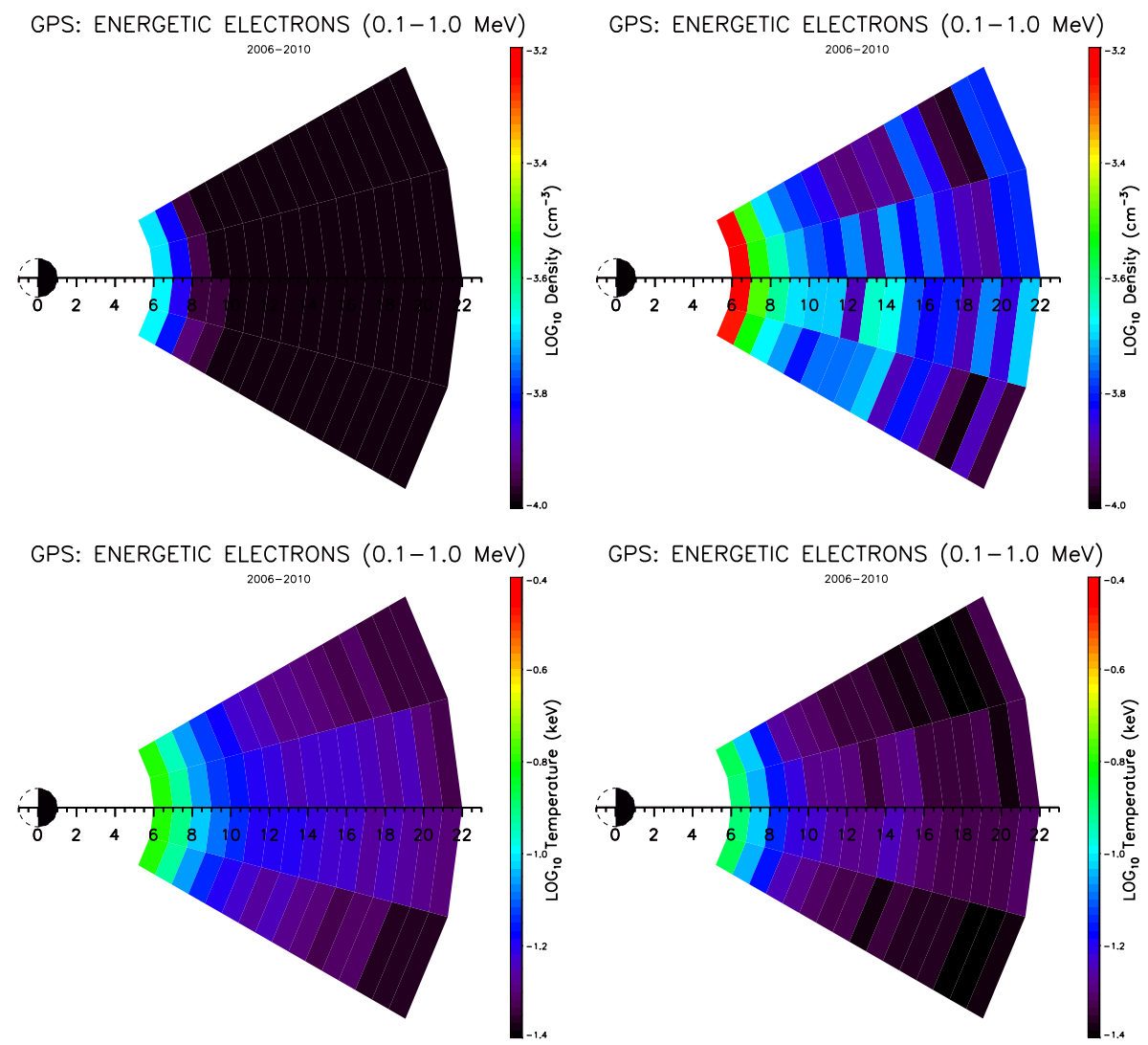

GPS: ENERGETIC ELECTRONS $(0.1-1.0 \mathrm{MeV})$
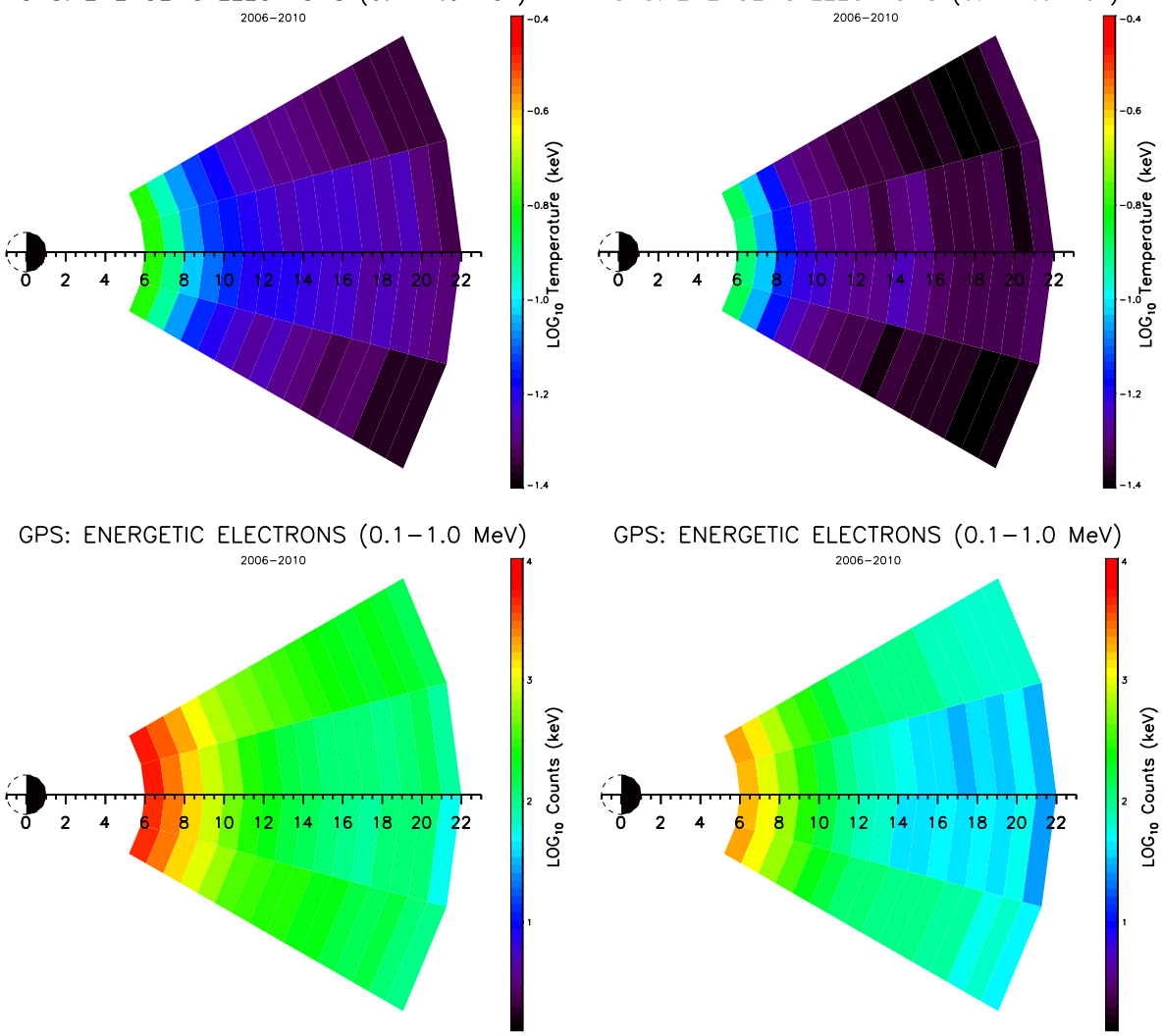

GPS: ENERGETIC ELECTRONS (0.1-1.0 MeV)

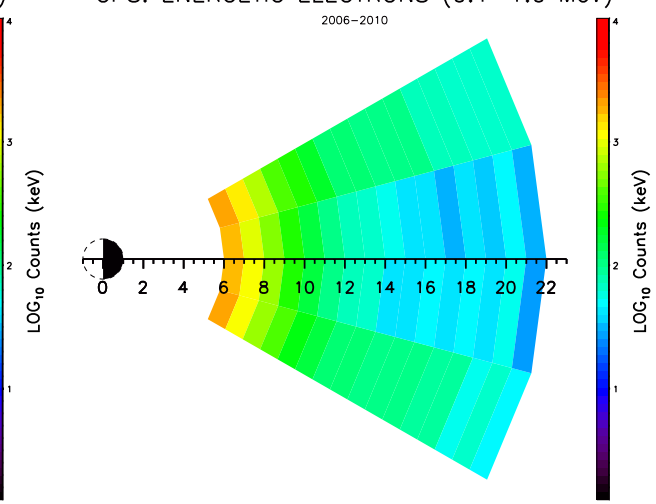

Fig. 4. Plots of the averaged energetic electron density ( $N$ : top row), temperature ( $T$ : middle row) and counts (bottom row) derived from measurements by GPS spacecraft at high-latitude and then mapped to the equatorial plane using the Tsyganenko T89 model as a function of LT and L for slow solar wind (left column: $V_{\mathrm{SW}}=100-400 \mathrm{~km} \mathrm{~s}^{-1}: 58857$ data points) and fast solar wind (right column: $V_{\mathrm{SW}}=500$ $1000 \mathrm{~km} \mathrm{~s}^{-1}: 23087$ data points).

2. The mean density of energetic electrons is correlated with Kp and with solar wind speed. The density of energetic electrons is higher during periods of increased magnetospheric convection, and during periods of fast solar wind. i.e. The strength of driving (e.g. by weak HSSs compared to strong HSSs) is an important factor for energetic electron density and temperature in the magnetotail (cf. Denton and Borovsky, 2011).
3. During periods of fast solar wind $\left(V_{\mathrm{SW}}=500\right.$ $1000 \mathrm{~km} \mathrm{~s}^{-1}$ ) the density of energetic electrons in the magnetotail is greater than during periods of slow solar wind $\left(V_{\mathrm{SW}}=100-400 \mathrm{~km} \mathrm{~s}^{-1}\right)$ by a factor of more than two. In contrast the temperature of energetic electrons is slightly higher during slow solar wind than during fast solar wind. 

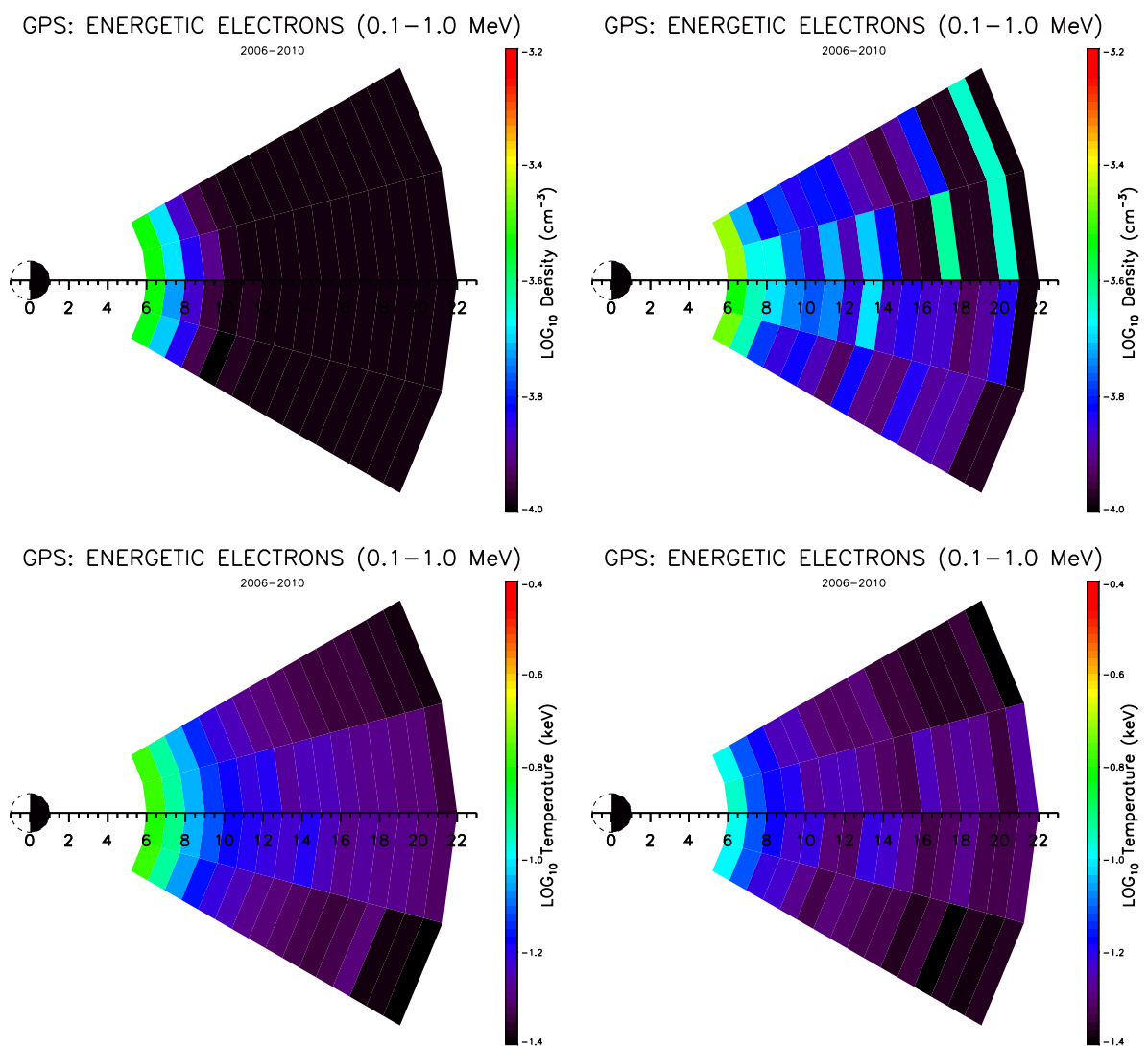

GPS: ENERGETIC ELECTRONS (0.1-1.0 MeV)

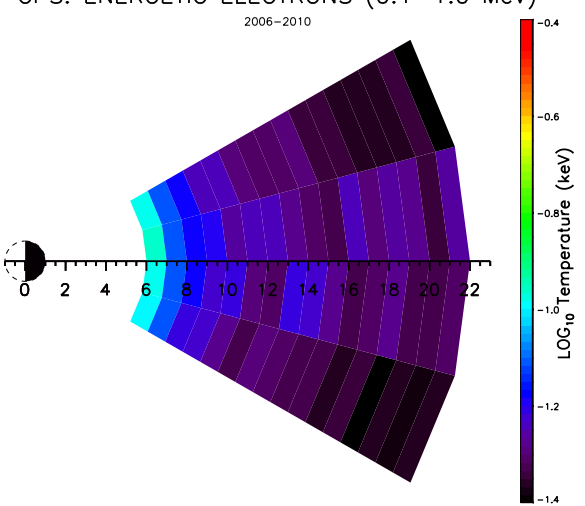

GPS: ENERGETIC ELECTRONS (0.1-1.0 MeV)

GPS: ENERGETIC ELECTRONS (0.1-1.0 MeV)
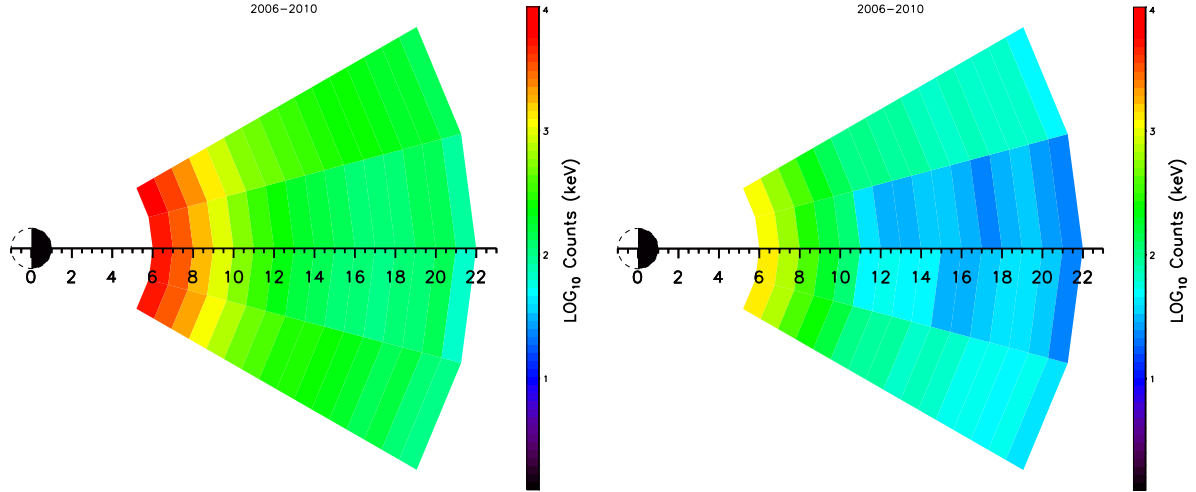

Fig. 5. Plots of the averaged energetic electron density ( $N$ : top row), temperature ( $T$ : middle row) and counts (bottom row) derived from measurements by GPS spacecraft at high-latitude and then mapped to the equatorial plane using the Tsyganenko T89 model as a function of LT and L for quiet geomagnetic conditions (left column: Kp=0-2: 66464 data points), disturbed geomagnetic conditions (right column: $\mathrm{Kp}>$ 3: 14095 data points).

We are currently utilising the database of GPS observations to study the energetic electron population in the magnetotail in relation to other plasmas in the magnetosphere (e.g. Borovsky and Cayton, 2011). Previous studies have revealed transport timescales from the solar wind to the inner magnetosphere during HSS-events (e.g. Denton and Borovsky, 2008). Future studies will follow a similar methodology to study links between plasmas in the solar wind and different regions of the magnetosphere and hence investigate the source and loss mechanisms for energetic electrons found in more-distant regions of the Earth's magnetotail. The current study contains data from 2006-2010 only - a reasonably quiet period during the declining phase of the solar cycle. As time progresses more and more data (eventually from a constellation of 24 or more GPS satellites) will be available for study, including more data from geomagnetically active periods. 
Acknowledgements. The authors would like to thank Evan Noveroske, custodian of the GPS data archive at LANL, for provision of the basic BDD and CXD data files, and the Space Physics Data Facility (SPDF) and National Space Science Data Center (NSSDC) for provision of the OMNI2 database. We thank Joe Borovsky, Reiner Friedel, John Steinberg and Joachim Birn for helpful discussions. Work at Lancaster was supported under grant ST/G002401/1 from the STFC. Work at LANL was supported by the US Department of Energy and the NASA Heliophysics TR\&T program. Parts of this work were carried out at Los Alamos National Laboratory during the autumn of 2010 and MHD wishes to thank Joe Borovsky for financial support and all at ISR-1 for hospitality during this visit.

Topical Editor R. Nakamura thanks one anonymous referee for her/his help in evaluating this paper.

\section{References}

Åsnes, A., Friedel, R. W. H., Lavraud, B., Reeves, G. D., Taylor, M. G. G. T., and Daly, P.: Statistical properties of tail plasma sheet electrons above $40 \mathrm{keV}$, J. Geophys. Res., 113, A03202, doi:10.1029/2007JA012502, 2008.

Baker, D. N. and Stone, E. C.: Energetic electron anisotropies in the magnetotail: Identification of open and closed field lines, Geophys. Res. Lett., 3, 557-560, 1976.

Borovsky, J. E. and Cayton, T. E.: Entropy mapping of the outer electron radiation belt between the magnetotail and geosynchronous orbit, J. Geophys. Res., 116, A06216, doi:10.1029/2011JA016470, 2011.

Borovsky, J. E. and Denton, M. H.: Electron loss rates from the outer radiation belt caused by filling of the outer plasmasphere: The calm before the storm, J. Geophys. Res., 114, A11203, doi:10.1029/2009JA014063, 2009.

Borovsky, J. E. and Denton, M. H.: A survey of the anisotropy of the outer electron radiation belt during highspeed-stream-driven storms, J. Geophys. Res., 116, A05201, doi:10.1029/2010JA016151, 2011.

Borovsky, J. E., Thomsen, M. F., McComas, D. J., Cayton, T. E., and Knipp, D. J.: Magnetospheric dynamics and mass flow during the November 1993 storm, J. Geophys. Res., 103, 2637326394, 1998.

Cayton, T. E., Belian, R. D., Gary, S. P., Fritz, T. A., and Baker, D. N., Energetic electron components at geosynchronous orbit, Geophys. Res. Lett., 16, 147-150, 1989.

Cayton, T. E., Drake, D. M., Spencer, K. M., Herrin, M., Wehenr, T. J., and Reedy, R. C.: Description of the BDD-IIR: Electron and Proton Sensors on the GPS. Technical Report LA-UR-98-1162, Los Alamos National Laboratory, Los Alamos, NM 87545, USA, 1998.

Cayton, T. E., Chen, Y., Friedel, R. H. W., and Kippen, R. M.: Analysis of Electron and Proton Environmental Data for MediumEarth Orbit (2000-present), Technical Report LA-UR-10-4234, Los Alamos National Laboratory, Los Alamos, NM 87545, USA, 2010.

Christon, S. P., Mitchell, D. G., Williams, D. J., Frank, L. A., Huang, C. Y., and Eastman, T. E.: Energy spectra of plasma sheet ions and electrons from $\sim 50 \mathrm{eV} / \mathrm{e}$ to $\sim 1 \mathrm{MeV}$ during plasma temperature transitions, J. Geophys. Res., 93, 2562-2572, 1988.
Christon, S. P., Williams, D. J., Mitchell, D. G., Huang, C. Y., and Frank, L. A.: Spectral characteristics of plasma sheet ion and electron populations during disturbed geomagnetic conditions, J. Geophys. Res., 96, 1-22, 1989.

Denton, M. H. and Borovsky, J. E.: Superposed epoch analysis of high-speed-stream effects at geosynchronous orbit: hot plasma, cold plasma and the solar wind, J. Geophys. Res., 113, A07216, doi:10.1029/2007JA012998, 2008.

Denton, M. H. and Borovsky, J. E.: Magnetosphere response to high-speed solar-wind streams: A comparison of weak and strong driving and the importance of extended periods of fast solar wind, J. Geophys. Res., in review, 2011.

Denton, M. H., Borovsky, J. E., and Cayton, T. E.: A densitytemperature description of the outer electron radiation belt during geomagnetic storms, J. Geophys. Res., 115, A01208, doi:10.1029/2009JA014183, 2010.

Distel, J. R., Blair, S. G., Cayton, T. E., Dingler, R., Guyker, F., Ingraham, J. C., Noveroske, E., Reedy, R. C., Spencer, K. M., and Wehne, T. J.: The Combined X-ray Dosimeter (CXD) on GPS Block IIR Satellites. Technical Report LA-UR-99-2280, Los Alamos National Laboratory, Los Alamos, NM, 87545, USA, 1999.

Fritz, T. A., Alothman, M., Battacharjya, J., Matthews, D. L., and Chen, J.: Butterfly pitch-angle distributions observed by ISEE-1, Planet. Space Sci., 51, 205-219, 2003.

Hilton, H.: L Parameter: A New Approximation, J. Geophys. Res., 76, 6952-6954, 1971.

Hones, E., Singer, S., and Rao, C. S. R.: Simultaneous observations of electrons $(\mathrm{E}>45 \mathrm{keV})$ at 2000-kilometer altitude and at 100,000 kilometers in the magnetotail, J. Geophys. Res., 73, 7339-7359, 1968.

Johnson, J. R. and Wing, S.: Northward interplanetary magnetic field plasma sheet entropies, J. Geophys. Res., 114, A00D08, doi:10.1029/2008JA014017, 2009.

King, J. H. and Papitashvili, N. E.: Solar wind spatial scales in and comparisons of hourly Wind and ACE plasma and magnetic field data, J. Geophys. Res., 110, A02104, doi:10.1029/2004JA010649, 2005.

Klida, M. M. and Fritz, T. A.: The Earth's magnetopause as a source and sink for equatorial nightside energetic charged particles, Ann. Geophys., 27, 4305-4316, doi:10.5194/angeo-274305-2009, 2009.

McIlwain, C. E. and Whipple, E. C.: The dynamic behavior of plasmas observed near geosynchronous orbit, IEEE Trans. Plasma Science, 14, 874-890, 1986.

Meng, C.-I.: Energetic electrons in the magnetotail at 60 Re, J. Geophys. Res., 76, 862-872, 1971.

Meng, C.-I., Lui, A. T. Y., Krimigis, S. M., Ismail, S., and Williams, D. J.: Spatial distribution of energetic particles in the distant magnetotail, J. Geophys. Res., 86, 5682-5700, 1981.

Montgomery, M. D.: Observations in the Earth's magnetotail by Vela Launch 2 Satellites, J. Geophys. Res., 73, 871-889, 1968.

Murayama, T. and Simpson, J. A.: Energetic electrons within the neutral sheet of the magnetospheric tail, J. Geophys. Res., 73, 891-905, 1968.

Retzler, J. and Simpson, J. A.: Relativistic electrons confined within the neutral sheet of the geomagnetic tail, J. Geophys. Res., 74, 2149-2160, 1969.

Sarris, T., Li, X., and Temerin, M.: Simulating radial diffusion 
of energetic $(\mathrm{MeV})$ electrons through a model of fluctuating electric and magnetic fields, Ann. Geophys., 24, 2583-2598, doi:10.5194/angeo-24-2583-2006, 2006.

Taylor, M. G. G. T., Reeves, G. D., Friedel, R. H. W., Thomsen, M. F., Elphic, R. C., Davies, J. A., Dunlop, M. W., Laakso, H., Lavraud, B., Baker, D. N., Slavin, J. A., Perry, C. H., Escoubet, C. P., Masson, A., Opgenoorth, H. J., Vallat, C., Daly, P. W., Fazakerley, A. N., and Lucek, E. A.: Cluster encounter with an energetic electron beam during a substorm, J. Geophys. Res., 111, A11203, doi:10.1029/2006JA011666, 2006.

Terasawa, T. and Nishida, A.: Simultaneous observations of relativistic electron bursts and neutral-line signatures in the magnetotail, Planet. Space Sci., 24, 855-866, 1976.
Tsyganenko, N. A.: A magnetospheric magnetic field model with a warped tail current sheet, Planet. Space Sci., 37, 5-20, 1989.

Tuszewski, M. G., Cayton, T. E., and Ingraham, J. C.: A New Numerical Technique to Design Satellite Energetic Electron Detectors, Nuclear Instruments and Methods in Physics Research, A482, 653-666, 2002.

Walker, R. J. and Farley, T. A.: Spatial distribution of energetic plasma sheet electrons, J. Geophys. Res., 77, 4650-4660, 1972.

Wing, S. and Newell, P. T.: Central plasma sheet ion properties as inferred from ionospheric observations, J. Geophys. Res., 103, 6785-6800, 1998. 